\title{
Bilirubin-induced neurologic dysfunction (BIND): appearances are fairly often deceptive
}

\author{
Lakatos $\mathrm{L}^{1 *}$ and Balla $\mathrm{G}^{2}$
}

${ }^{1}$ University of Debrecen, Faculty of Medicine, Department of Pediatrics, 4032 Debrecen, Nagyerdei krt. 1, Hungary

${ }^{2}$ Member of the Hungarian Academy of Science, University of Debrecen, Faculty of Medicine, Department of Pediatrics, 4032 Debrecen, Nagyerdei Krt. 1, Hungary

\begin{abstract}
On the basis of abundant research data and hypotheses in the literature, according to our concept, BIND is a neurodegenerative disease (ND) of immature brain caused by accumulation of free metals and unconjugated bilirubin (UCB), and UCB-Cu complex (as prooxidant), respectively, in the basal ganglia (BG) and other parts of central nervous system (CNS) relevant to BIND. The main reason is the hemolysis of neonatal red blood cells. In this way a great amount of heavy metals are liberated together with accumulation of reactive oxygen species (ROS). The circulated elements in the bloodstream can pass through the immature blood-brainbarrier (BBB), finding entrance into CNS. In addition, ROS increases BBB permeability creating a dangerous vitious circle in the neonatal brain, especially in the basal ganglia (BG). Bilirubin staining of BG and the neurotoxic effects of bile pigment in CNS (which were studied almost exclusively as a background of kernicterus in the last century) constitute only apparently the principal factor of bilirubin encephalopathy.
\end{abstract}

\begin{abstract}
Abbreviations: AD: Alzheimer's Disease; BG: Basal Ganglia; BBB: Blood Brain Barrier; BIND: Bilirubin-Induced Neurologic Dysfunction; Cp: Ceruloplasmin; CNS: Central Nervous System; D-PA: D-Penicillamine; MD: Metal Dyshomeostasis; MT: Metallothionein; NHBI: Neonatal Hyperbilirubinemia; ND: Neurodegenerative Disease; PD: Parkinson Disease; ROS: Reactive Oxygen Species; ROP: Retinopathy of Prematurity; WD: Wilson disease.
\end{abstract}

\section{Introduction}

More than 8 million Americans and 450 million peoples across the globe are affected by neurological disorders (including birth defects and BIND). The economic burden of these diseases is $\$ 2014$ billion per year. For developing the new research incites among the researchers their valuable work is really important to neurology [1]. Very wideranging studies have long been made on the possible biochemical transformations of UCB which is formed during the decomposition of hemoglobin. Particular attention has been paid to its photochemical and redox reactions but the relevant publications comprise only a very small proportion of those dealing with the molecular biochemistry of $\mathrm{UCB}$ and metal interactions [2].

Neonatal hyperbilirubinemia (NHBI) is a common condition in the first week of postnatal life. In the past decades, interest in bilirubin damage of the brain has been reawakened by an increase in its prevalence, owing to failure to closely observe infants discharged from the hospital well before the peak of NHBI. There is a tremendous variability in babies' vulnerability toward UCB for reasons not yet explained, but preterm birth, sepsis, hypoxia, hypoperfusion, hyperosmolality, acidosis, hypalbuminemia and hemolytic disease et cet. (underlying diseases or comorbidities) are comprised as risk factors, so, the UCB levels and neurological abnormalities are not strictly correlated. Subtle UCB damage may be responsible for many more cases of: learning disabilities, central auditory processing disorders, dyslexia, oculomotor dyspraxia, movement disorders, and autism spectrum disease (ASD), and may even predispose to Parkinson's disease (PD) or schizophrenia in adulthood [3]. The pathomechanisms of BIND have not been fully understood yet. The mechanisms of UCB neurotoxicity are still also unclear, and little is known about lasting sequelae attributable to NHBI. Our hypothesis addresses the medical necessity of chelation therapy (with D-PA) in the neonatal period [4] as it is feasible that UCB molecule reviels particular affinity to copper stored in BG of the neonatal brain, where copper-bilirubin complex can be formed together with the production of hydroxyl radical $\left(\mathrm{OH}^{-}\right)$. In addition, various amount of free metal ions can be accumulated in the intravascular space and in the tissues (especially in BG) during hemolytic processes.

\section{Discussion}

\section{Pathological basal ganglia activity [5]}

The BG represent large subcortical nuclear masses. It is agreed that core components comprise the caudate nucleus, the nucleus accumbens, the putamen, and the globus pallidus. The caudate nucleus and putamen together are sometimes called the striatum, and the putamen and globus pallidus are together sometimes described as the lentiform nucleus. Functionally, BG has considerable connections to the cerebral cortex, thalamus, and brain stem; so, anatomists consider portions of the thalamus as components of BG. A literature review was aimed at assisting us (as pediatricians) to provide further understanding with bilateral symmetrical BG and thalamic lesions on magnetic resonance imaging (MRI). The high-signal-intesity lesions on T2-weighted images can be caused by edema, gliosis, demyelinization, neuronal necrosis, or cystic degeneration both in WD and BIND.

Correspondence to: Lajos Lakatos, University of Debrecen, Faculty of Medicine, Department of Pediatrics, 4032 Debrecen, Nagyerdei krt. 1, Hungary; E-mail: lakatosl@kenezykorhaz.hu.

Key words: Copper Toxicity; Bilirubin-Induced Neurologic Dysfunction; Oxidative Stress; Copper-Bilirubin Complex; D-Penicillamine in the neonatal period

Received: August 28, 2017; Accepted: September 15, 2017; Published: September 18, 2017 


\section{Role of metals and oxidative stress in the human neurodegenerative and neurodevelopmental disorders [6]}

The brain (mostly the BG) accumulates among the highest levels of transition metals in the body for normal function, including redoxactive copper. This high-redox metal load, in combination with the brain disproportionately active oxygen metabolism, makes this organ particularly susceptible to oxidative stress [7]. Metal ions such as calcium, zinc, iron and copper are key players in brain neurobiology; their homeostasis is altered in most ND conditions. The metal dyshomeostasis (MD) in the brain and related organs, and loss of the strict regulation is implicated in neurotoxic stress and in a variety of NDs including BIND and prion mediated encephalopathies and other diseases $[8,9]$. Pathologic changes to the CNS in these disorders are always associated with a significant dyshomeostasis of tissue metals (particularly copper). Excess copper may react upon sulfhydryl, carboxyl, or amine groups, resulting in improper enzymatic activity or damage to cellular structure. Despite the ubiquitous presence of toxic copper within the brain, pathologic findings are limited primarily to the BG, thalamus, and brain stem. Histopathologic studies have shown abnormalities throughout this system in patients suffering from MD. These abnormalities include atrophy, spongy softening, cavitation, a general reduction of neurons, increased cellularity, and the presence of characteristic particles (Opalski, Lewy bodies). The pathologic changes result in an increased amount of extracellular copper which causes oxidative stress and cell destruction. Many diseases of the BG have some disorder of movement as their primary symptom, ranging from an excess of (abnormal) involuntary movements such as in chorea to a poverty and slowness of movement as in PD, Alzheimer disease (AD) and WD as illustrated in several clinical cases and UCB encephalopathy where a characteristic yellow staining can be observed in fresh or frozen sections of the brain obtained within 7-10 days after the initial bilirubin insult. If the affected infant survives the neonatal period and subsequently dies, the yellow staining may no longer be present, but the BG will display microscopic evidence of cell injury, neuronal loss, and glial replacement. Newborns, especially preterm infants, are particularly vulnerable to reactive ROS because they exhibit accelerated production of free radical and limited antioxidant protection, which increases the susceptibility of rapidly growing tissues to damage. There is now a large body of literature demonstrating that free or weakly bound iron and copper ions may exert their toxic action on BG. Predominantly the cellular content of copper determines copper-induced toxicity in brain astrocytes .

\section{Potential molecular mechanisms of bilirubin-induce neurologic dysfunction}

The questions arise: (1) How to call UCB: friend or foe? (2) If the bilirubin is really an „enemy”, how does it induce its dangerous effects?

\section{Ad (1) The exact UCB concentration associated with kernicterus}

in the healthy term infant is unpredictable. In a Danish popolationbased study, the neonates with total serum bilirubin levels of $\geq 25$ $\mathrm{mg} / \mathrm{dL}$ didn't show any neurologic dysfunctions at 5 years of follow up [10]. Bilirubin, which is derived from its metabolic precursor biliverdin, is the end product of heme catabolism. It has been proposed that UCB is an excellent endogen antioxidant present in human extracellular fluids [11]. Bilirubin can suppress oxidation of lysosomes at oxygen concentrations that are physiologically relevant. It can act as an important cytoprotector of tissues that are poorly equipped with antioxidant defense systems, including myocardium and nervous tissue. Higher UCB concentrations resulted in lower lipid hydroperoxide levels. Therefore, we think that UCB in itself is actually our friend, that is: Bilirubin, The Gold Within [12].

\section{Ad (2) Toxic Side of Bilirubin}

Erythrocyte morphological changes have been seen with incubation of cells with different molar ratios of UCB. These changes occur as the bilirubin/human serum albumin molar ratio increases. This indicates that bilirubin can illicit toxicity in the erythrocyte membrane in a concentration and temperature-dependent manner, causing hemolysis [13]. Several studies have found that NHBI is associated with higher risk of movement disorder and, even more, developmental delay. The management dilemma for a clinician is that UCB is a beneficial antioxidant at low (and may be at moderatly higher) levels, but a neurotoxin at $>20 \mathrm{mg} / \mathrm{dL}$ levels ("vigintophobia" [14]), where it can impair the normal developmental maturation of the neonatal brain.

\section{Copper homeostasis in infant [15]}

Copper is essential for the normal growth and development of human fetuses, infants, and children and it is crucial for the normal development of the brain which has among the highest levels of copper, as well as iron and zinc, in the body. Copper is an interesting essential micronutrient. Deficiency and excess intake both induce a variety of clinical manifestations affecting mainly the hematopoietic system, the skeleton, the liver, and the brain. Although copper transport to the fetus is high and liver storage is efficient, copper export from the hepatocytes to the bile and to blood ceruloplasmin $(\mathrm{Cp})$ are reduced during this stage of life because of liver function immaturity. This leads to a high copper accumulation in the liver and brain, in a magnitude similar to that observed in WD. The increased liver and brain copper storage of the fetus may have a selective evolutionary advantage since it may prevent copper deficiency during the first months of life when the child receives a relatively low copper supply from breast milk [16].

\section{Metal regulatory proteins in the neonatal period $[17,18]$}

A variety of proteins are involved in the regulation of metal metabolism and the oxidative response and many are involved in iron or copper metabolism due to the redox activity of those metals. Protein misfolding and conformational changes are also a cornerstone of NDs. All metals with known physiological functions are bound by albumin [19]. A decrease in metal binding of albumin means more free metal available to produce oxidative stress and other physiological effects such as influence of calcium $(\mathrm{Ca}++)$ homeostasis by altering the conformational structure of the pumps, enzymes, binding proteins, and channels that regulate $\mathrm{Ca}++$ flow. Often, this results in elevating free intracellular $\mathrm{Ca}++$ levels which may produce depletion of glutathione/ GSH with a downstream induction of DNA damage and eventual cell death [20]. Therefore, the bilirubin-mediated neurotoxicity is partly due to increased rate of cell apoptosis and higher levels of intracellular free $\mathrm{Ca}++$ ion level. $\mathrm{Cp}$ is a large blood protein synthesized by the liver with the primary role of transporting copper. If a disease process (e.g. hepatic failure) or insufficient synthesis in the neonatal period lowers the production of $\mathrm{Cp}$, the free copper would increase and copper mediated oxidative stress would be enhanced. In addition, there is some evidence that under oxidative stress conditions Cp may induce further oxidative stress in a manner akin to a positive feedback mechanism [21]. Copper transporter 1 (Ctr1) has a high affinity for copper and serves to transport copper into the interior of the cell. It is not highly expressed in the brain, where the choroid plexus may contain the greater proportion. The lower levels of expression in the brain, however, should not be taken as a sign that $\mathrm{Cu}$ metabolism is not important in the brain as several neural pathologies $(\mathrm{AD}$, spongiform encephalopathies) have been linked to disordered copper metabolism 
[22]. Metallothionein (MT) is a cysteine rich protein involved in the regulation of zinc and other metals (mainly copper, and selenium). This protein is found in a variety of forms (I-IV) in mammals, and MTII are the most abundant in the CNS where MT is found mostly in astrocytes. MT plays an important role in cell signaling. Neonatal brain has lower MT concentrations than adult brain, increasing to adult levels by Day 21 [23]. Elucidating the role of the metallochaperone Atox1, it is obvious that Atox1-deficient cells accumulates high levels of intracellular copper, and metabolic studies indicate: this defect originated from the impaired cellular copper efflux. These data reveal a direct role for Atox 1 in trafficking of intracellular copper to the secretory pathway of mammalian cells and demonstrate that metallochaperone plays a critical role in perinatal copper homeostasis [24].

\section{Copper and other metal toxicity syndrome [25]}

Copper toxicity is a condition that is increasingly common in this day and age, due to the widespread occurrence of copper in our food, copper fungicides, e-cigs, copper IUD's, hot water pipes, along with the common nutritional deficiencies in zinc, manganese and other trace minerals that help keep levels of copper in balance. Copper is a very stimulating mineral to the nerves and CNS. Its effects on neurotransmitter levels can give rise to many psychological imbalances such as mood swings, depression, mental agitation, feeling overstimulated, restlessness, anxiety and insomnia. A number of the toxic effects of copper comes from its ability to accept and donate single electrons as it changes oxidation state. This increase in unmediated ROS is generally termed oxidative stress. When women become pregnant, their estrogen levels rise, greatly increasing the retention of copper in the body. This metal will pass through the placenta into the unborn child. So many children are being born with toxic levels of copper and other heavy metals which were stored in the mother's body (BOX 1).

A recent report by the National Research Council found that $50 \%$ of all pregnancies in the US are now resulting in prenatal or postnatal mortality, significant birth defects, developmental neurological problems, or otherwise chronically unhealthy babies.

\section{BOX 1. Many children are being born with toxic levels of} heavy metals [26]

Studies have also found heavy metals to deplete glutathione and bind to protein-bound sulfhydryl groups, resulting in inhibiting $\mathrm{SH}$-containing enzymes and production of reactive oxygen species such as superoxide ion, hydrogen peroxide, and hydroxyl radical. Here, it is noteworthy, how prominent similarities exist between WD and neonates with kernicterus concerning the copper metabolism, the neuropsychiatric manifestations and the hystopathological findings (BOX 2).

\section{NEUROPSYCHIATRIC MANIFESTATIONS}

WD: Movement disorders, tremors, involuntary movements, choreoathetosis, dysarthria, dystonia, personality changes, uncontrolled emotional outbursts

Kernicterus: generalized dystonia, athetoid cerebral palsy, paralysis of upward gaze, sensori- neural hearing loss.

BIND: impairment of audiologic, speech, and language processing as well as disturbances in visual-motor and cognitive functions associated with failure of fine neuromotor control (extrapyramidal signs

At AUTOPSY (both in WD and kernicterus): marked neuronal loss with demyelination and astrocytic replacement [27].

\section{BOX 2. Common neuropsychiatric manifestations and pathological findings in WD and bilirubin encephalopathy}

In the neonatal period the ability of the liver to synthesize $\mathrm{Cp}$ is not fully developed and adult levels of the protein are not found in the blood till about three months of age. It is interesting therefore that the infant liver has a very much higher copper content than is found in the adult and a fall in concentration does not takes place until the ability to synthesize $\mathrm{Cp}$ has fully developed.

\section{New concept for development of bind}

Very wide-ranging studies have long been made on the possible biochemical transformations of UCB, which is formed during the decomposition of haemoglobin. Particular attention has been paid to its photochemical and redox reactions [2] but the relevant publications comprise only a very small proportion of those dealing with the molecular biochemistry of UCB and metals interactions. UCB has a special affinity for the globus pallidus, the hippocampus, and the subthalamic nucleus because they are also target brain regions for divalent metals ( $\mathrm{Cu}, \mathrm{Fe}, \mathrm{Zn}$ et cet.). Neurodegeneration: a return to immaturity [25]? This question certainly arouses the attention of neonatologists as the immature and strikingly vulnerable neurons play important role in the pathogenesis of BIND. The increased vulnerability of premature infants to brain damage may be due to a proneness of immature nerve cells to toxic stimulus. The developing neurons undergo programmed cell death, a necessary phenomenon for proper nervous system development. Following the developmental period, neurons mature and restrict the apoptotic pathway to permit long-term survival. On the basis of above described abundant research data and hypotheses, according to our concept, the BIND is an ND of immature brain caused by accumulation of free metals and UCB-Cu complex (as prooxidant) in the BG and other parts of CNS relevant to BIND. In strictly biological terms, at least 3 loci exist where UCB and copper can be "fusioned” in the neonatal period: (1) during hemolysis high UCB and copper level can be developed in the blood; (2) one albumin can bind one $\mathrm{Cu}^{++}$in the primary binding site. At higher concentration of copper (if possible under certain conditions), loosely bound atoms, and can be very easily taken out by UCB. The bile pigment itself can displace loosely bound copper ions, which are electrostatically attached due to high negative charge on the surface of albumin; (3) in the basal ganglia [27]. The main comorbidity is the hemolysis of neonatal blood red cells. During this process a great amount of heavy metals (mainly iron and copper) may circulate in free form in the bloodstream, and can pass through the blood-brain-barrier (BBB), finding entrance into the CNS as well. Understanding the differences between neonatal and adult erythrocytes is critical in the evaluation of perinatal erythrocyte disorders. The reason for the reduced red blood cell survival observed in newborns is not known, although there are many biochemical differences between adult and neonatal red blood cells (RBCs). Increased oxidant sensitivity of newborn RBCs and relative instability of fetal hemoglobin have been considered as possible causes for this shortened lifespan.

\section{Conclusion}

The basic role of metal ions in neurological pathologies is generally accepted, - except for the case of BIND. Free copper ion in itself or binding to UCB and forming metal-bilirubin complex(es) involved in neurologic dysfunction, therefore they are important factors for whole brain damage processes in BIND [28]. We believe it so that, on the base of evidence written above, UCB cannot be considered for "Evil one” in 


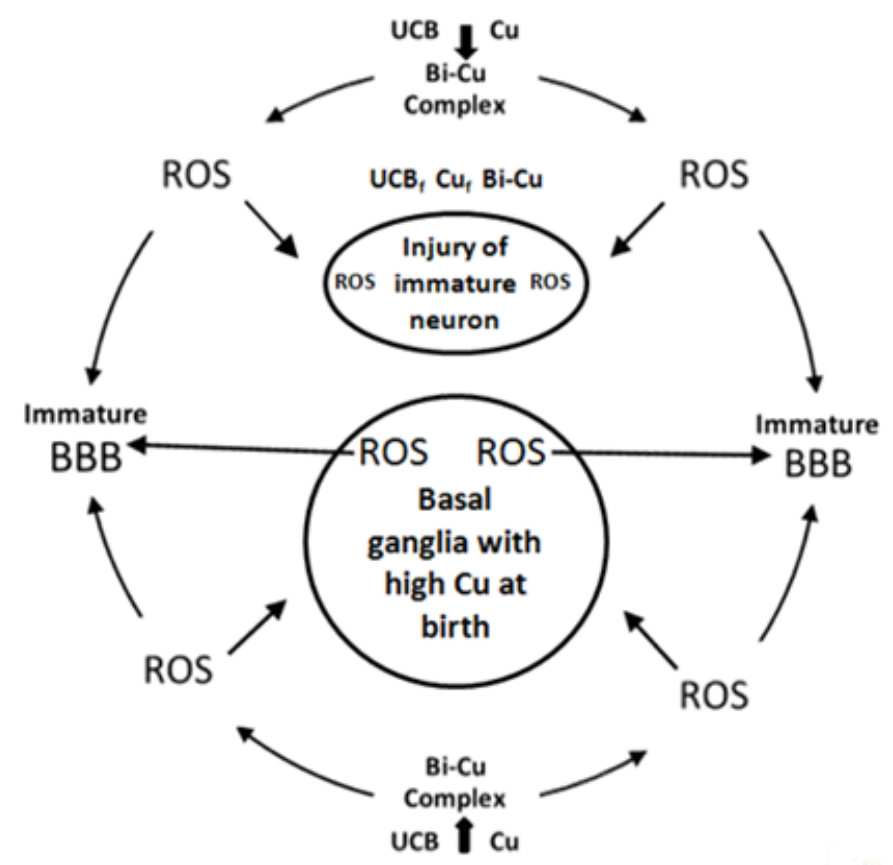

Figure 1. Molecular mechanism of BIND.

(UCB $=$ unconjugated bilirubin, $\mathrm{UCB}_{\mathrm{f}}=$ free unconjugated bilirubin, $\mathrm{Cu}_{\mathrm{f}}=$ free $\mathrm{Cu}, \mathrm{ROS}=$ reactive oxygen species, $\mathrm{BBB}=$ blood brain barrier)

the etiology of NHBI in the neonatal period. (Figure 1) demonstrates our concept about the chronic bilirubin encephalopathy.

We hope that our theory will help answer some of the unsolved questions and concerns ocurred in the etiology and pathomechanisms of BIND. The beneficial neuropharmacological actions of metal-targeted (chelating) agents most likely arise from local metal redistribution rather than from massive metal removal. [29]. The chelation therapy for non-metal overload indications continues to be investigated. Our present review address the medical necessity of the use of a chelating agent (D-PA) in the neonatal period (mainly for the prevention or treatment of BIND and ROP) [30].

\section{References}

1. Oliver F (2017) Personal communication.

2. Hansen TW (2016) Biology of Bilirubin Photoisomers. Clin Perinatol 43: 277-290. [Crossref]

3. Maisels MJ, Newman TB (1998) Jaundice in full-term and near-term babies who leave the hospital within 36 hours. The pediatrician's nemesis. Clin Perinatol 25: 295-302. [Crossref]

4. Balla G, Lakatos L, Vekerdy-Nagy Z (2015) Chelation therapy in the neonatalperiod: D-Penicillamine can exert neuroprotective effects in kernicterus and retinopathy of prematurity. IJPSR 6: 4269-4276.

5. Wichmann T, Dostrovsky JO (2011) Pathological basal ganglia activity in movement disorders. Neuroscience 198: 232-244. [Crossref]

6. Barnham KJ, Bush AI (2014) Biological metals and metal-targeting compounds in major neurodegenerative diseases. Chem Soc Rev 43: 6727-6749. [Crossref]

7. Rivlin-Etzion M1, Marmor O, Saban G, Rosin B, Haber SN, et al. (2008) Low-pass filter properties of basal ganglia cortical muscle loops in the normal and MPTP primate model of parkinsonism. $J$ Neurosci 28: 633-649. [Crossref]

8. Marseglia L, D’Angelo G, Manti S, Arrigo T, Barberi I, et al. (2014) Oxidative stressmediated aging during the fetal and perinatal periods. Oxid Med Cell Longev 2014: 358375. [Crossref]
9. Bulcke F, Santofimia-Castaño P, Gonzalez-Mateoset A, Dringen R (2015) Modulation of copper accumulation and copper-induced toxicity by antioxidants and copper chelators in cultured primary brain astrocytes. J Trace Elem Med Biol 32: 168-176. [Crossref]

10. Vandborg PK, Hansen BM, Greisen G, Jepsen M, Ebbesen F (2012) Follow-up of neonates with total serum bilirubin levels â\%o $25 \mathrm{mg} / \mathrm{dL}$ : a Danish population-based study. Pediatrics 130: 61-66. [Crossref]

11. Stocker R, Yamamoto Y, McDonagh AF, Glazer AN, Ames BN (1987) Bilirubin is an antioxidant of possible physiological importance. Science 235: 1043-1046. [Crossref]

12. Seppen J, Bosma P (2012) Bilirubin, the gold within. Circulation 126: 2547-2549. [Crossref]

13. Bhutani VK, Wong R2 (2015) Bilirubin-induced neurologic dysfunction (BIND). Semin Fetal Neonatal Med 20: 1. [Crossref]

14. Watchko JF, Oski FA (1983) Bilirubin $20 \mathrm{mg} / \mathrm{dL}=$ vigintiphobia. Pediatrics 71: 660663. [Crossref]

15. Olivares M, Araya M, Uauy R (2000) Copper homeostasis in infant nutrition: deficit and excess. J Pediatr Gastroenterol Nutr 31: 102-111. [Crossref]

16. de Romaña DL, Olivares M, Uauy R, Araya M (2011) Risks and benefits of copper in light of new insights of copper homeostasis. J Trace Elem Med Biol 25: 3-13. [Crossref]

17. Oettl K, Stauber RE (2007) Physiological and pathological changes in the redox state of human serum albumin critically influence its binding properties. $\mathrm{Br} J$ Pharmacol 151: 580-590. [Crossref]

18. Simms BA, Zamponi GW (2012) Trafficking and stability of voltage-gated calcium channels. Cell Mol Life Sci 69: 843-856. [Crossref]

19. Paradis M, Gagn'e J, Mateescu MA (2010) The effects of nitric oxide-oxidase and putative glutathione peroxidase activities of ceruloplasmin on the viability of cardiomyocytes exposed to hydrogen peroxide. Free Rad Biol Med 49: 2019-2027. [Crossref]

20. Hamza I, Faisst A, Prohaska J, Chen J, Gruss P, et al. (2001) The metallochaperone Atox1 plays a critical role in perinatal copper homeostasis. Proc Natl Acad Sci U S A 98: 6848-6852. [Crossref]

21. Bulcke F, Santofimia-Castaño P, Gonzalez-Mateos A (2015) Modulation of copper accumulation and copper-induced toxicity by antioxidants and copper chelators in cultured primary brain astrocytes. J Trace Elem Med Biol 32: 168-176. [Crossref]

22. Peters T, Jr. (1995) All about Albumin. 3 - Ligand Binding by Albumin.Biochemistry, Genetics, and Medical Applications. ISBN: 978-0-12-552110-9; 76-132.

23. Wilson L (2015) Copper toxicity syndrome. (C) Revised, September 2015 The Center For Development.

24. Parashari UC, Singh R, Yadav R, Aga P (2009) Changes in the globus pallidus in chronic kernicterus. J Pediatr Neurosci 4: 117-119. [Crossref]

25. Kole AJ, Annis RP, Deshmukh M (2013) Mature neurons: equipped for survival. Cell Death Dis 4: e689. [Crossref]

26. Reproductive and Developmental Toxicity of Metals Ed by Clarkson TW, Nordberg GF, Sager PR (1983) New York Plenum Press · New York and London

27. Adhikari S, Joshi R, Gopinathan C (1998) Bilirubin as an anti precipitant against copper mediated denaturation of bovine serum albumin: formation of copper-bilirubin complex. Biochim Biophys Acta 1380: 109-114. [Crossref]

28. Lutsenko S, Bhattacharjee A, Hubbard AL (2010) Copper handling machinery of the brain. Metallomics 2: 596-608. [Crossref]

29. Mot AI, Wedd AG, Sinclair L, Brown DR, Collins SJ, et al. (2011) Metal attenuating therapies in neurodegenerative disease. Expert Rev Neurother 11: 1717-1745. [Crossref]

30. Lakatos L, Balla G, Pataki I (2017) Copper-induced oxidative/nitrosative stress and excitoxicity in the neonatal period: neuroprotection with D-Penicillamine. Pediatric Dimensions 2: 1-6.

Copyright: C2017 Lakatos L. This is an open-access article distributed under the terms of the Creative Commons Attribution License, which permits unrestricted use, distribution, and reproduction in any medium, provided the original author and source are credited. 